\title{
Critical Care Workers Have Lower Seroprevalence of SARS-CoV-2 IgG Compared with Non-patient Facing Staff in First Wave of COVID19
}

\author{
Helen E. Baxendale ${ }^{1 *}$, David Wells ${ }^{2}$, Jessica Gronlund ${ }^{1}$, Angalee Nadesalingham ${ }^{2}$, Mina \\ Paloniemi², George Carnell2 ${ }^{2}$, Paul Tonks², Lourdes Ceron-Gutierrez ${ }^{3}$, Soraya Ebrahimi ${ }^{3}$, \\ Ashleigh Sayer ${ }^{3}$, John A.G. Briggs ${ }^{4}$, Xiaoli Ziong ${ }^{4}$, James A Nathan², Guinevere Grice ${ }^{2}$, Leo \\ C James ${ }^{4}$, Jakub Luptak ${ }^{4}$, Sumita Pai ${ }^{1}$, Jonathan L Heeney ${ }^{2}$, Sara Lear ${ }^{3}$, Rainer Doffinger ${ }^{3}$ \\ ${ }^{1}$ Royal Papworth Hospital NHS Foundation Trust Cambridge, UK \\ 2 University of Cambridge, UK \\ ${ }^{3}$ Cambridge University Hospitals NHS Foundation Trust, UK \\ ${ }^{4}$ MRC Laboratory of Molecular Biology, UK
}

\begin{abstract}
Introduction: In early 2020, at first surge of the coronavirus disease 2019 (COVID-19) pandemic, many health care workers (HCW) were re-deployed to critical care environments to support intensive care teams looking after patients with severe COVID-19. There was considerable anxiety of increased risk of COVID-19 for these staff. To determine whether critical care HCW were at increased risk of hospital acquired infection, we explored the relationship between workplace, patient facing role and evidence of immune exposure to the severe acute respiratory syndrome coronavirus 2 (SARS-CoV-2) within a quaternary hospital providing a regional critical care response. Routine viral surveillance was not available at this time. Methods: We screened over $500 \mathrm{HCW}$ ( $25 \%$ of the total workforce) for history of clinical symptoms of possible COVID19, assigning a symptom severity score, and quantified SARS-CoV-2 serum antibodies as evidence of immune exposure to the virus. Results: Whilst $45 \%$ of the cohort reported symptoms that they consider may have represented COVID-19, 14\% had evidence of immune exposure. Staffs in patient facing critical care roles were least likely to be seropositive (9\%) and staff working in non-patient facing roles most likely to be seropositive (22\%). Anosmia and fever were the most discriminating symptoms for seropositive status. Older males presented with more severe symptoms. Of the 12 staff screened positive by nasal swab (10 symptomatic), 3 showed no evidence of seroconversion in convalescence. Conclusions: Patient facing staff working in critical care do not appear to be at increased risk of hospital acquired infection however the risk of nosocomial infection from non-patient facing staff may be more significant than previous recognised. Most symptoms ascribed to possible COVID-19 were found to have no evidence of immune exposure however seroprevalence may underrepresent infection frequency. Older male staff were at the greatest risk of more severe symptoms.
\end{abstract}

Keywords: COVID-19, SARS-CoV-2, health care workers, critical care, seroprevalence

Received: 21 December 2020 / Accepted: 28 May 2021

\section{INTRODUCTION}

In December 2019, clusters of patients with novel pneumonia-like symptoms were identified in Wuhan, China [1]. This disease is now known as coronavirus disease 2019 (COVID-19) and is caused by the severe acute respiratory syndrome coronavirus 2 (SARS$\mathrm{CoV}-2)$. Since the initial cases, the virus has so far in- fected over 35 million people causing over 1,000,000 deaths globally [2]. In response to this pandemic many countries have and continue to employ social distancing practices and for many working from home has become the norm for desk-based employments.

The risk of infection in HCWs is increased due to limited opportunity to comply with social distancing measures due to the need for patient contact. In 
addition, patients with severe disease are likely to be particularly infectious with high viral load at hospital admission $[3,4]$. Disease severity is also likely to be increased in HCWs as there is a high representation of members from Black, Asian and other Minority Ethnic groups (BAME) [5], noting that individuals identifying as BAME appear to be at increased risk of severe COVID-19 [6]. Guidelines for managing HCW exposure to SARS-CoV-2 are now modified to take account of ethnicity risk additional to previously acknowledged risk factors such as age, sex and co-morbidities [7]. Previous data has demonstrated that HCWs in patient facing roles, particularly 'front door' roles, are at increased risk of severe COVID-19 compared with nonpatient facing roles and the general population however the risk to critical care workers is less clear [4].

Early in the pandemic, access to virological detection of infection by polymerase chain reaction (PCR) was limited and diagnosis of infection was generally made on the basis of clinical presentation of symptoms that were considered consistent with possible SARSCoV-2 infection. Serological assays were developed to understand prevalence of infection in the population.

The primary aim of this study was to determine by serological assays whether staff working in a UK critical care and extracorporal membrane oxygenation (ECMO) centre (including ECMO retrieval staff), from April to June 2020, had evidence of greater exposure to SARS-CoV-2 than non-critical care and non-patient facing staff. The secondary aims were to determine whether reported symptoms of possible COVID-19 predicted serological evidence of exposure and whether certain staff groups by demographic, were more at risk of severe COVID-19.

\section{METHODS}

\section{Recruitment}

The study was approved by Research Ethics Committee Wales, IRAS: 96194 12/WA/0148. Amendment 5. Staff from Royal Papworth Hospital NHS Foundation Trust (RPH) were recruited through staff email over the course of 2 months: $20^{\text {th }}$ April 2020-10th June 2020, as part of a prospective study to establish seroprevalence and immune correlates of protective immunity to SARS-CoV-2. All participants provided written, informed consent prior to enrolment in the study.

\section{Demographic details}

Information including age (in years), sex, ethnicity, job classification (critical/non-critical care and patient/ non-patient facing), history of chronic illness and possible exposure to COVID-19 patients was collected.

\section{COVID-19 history: symptom reporting and classifica- tion}

History of symptoms consistent with COVID-19 at any time from 1st January 2020 was recorded by questionnaire. COVID-19 symptoms were described in free text and then tabulated according to the following: anosmia, cough, dyspnoea, fatigue, fever, gastrointestinal (GI) disturbance, headache, myalgia, pharyngitis, upper respiratory infection (URTI). Symptom severity classes were classified according to WHO as 1 (asymptomatic), 2 (mild), 3-4 (moderate-severe) [8]. Staff with dyspnoea additional to cough and fever were classified as moderate and staff requiring oxygen were classified as severe disease severity.

History of previous swab PCR confirmed SARSCoV-2 infection was also collected. Symptomatic staff screening by nasopharyngeal swab commenced week starting $6^{\text {th }}$ April 2020 and a point prevalence NHS screening initiative of asymptomatic staff using a Roche SARS-CoV-2 Rapid Antigen Test was undertaken on the week starting $1^{\text {st }}$ June 2020.

\section{Serological Assessment}

Blood was taken and serum isolated for analysis of IgG binding to SARS-CoV-2 Spike (S), Nucleocapsid (N) and Receptor Binding Domain (RBD) proteins using a Luminex based multiplexed particle flow cytometry assay developed at Addenbrookes Hospital NHS Trust, and which has been accredited by the UK national accreditation body (UKAS, https://www.ukas.com/). To classify samples as seropositive or seronegative based on Luminex readings of SARS-CoV-2 N, S, and S-RBD binding, pre-pandemic sera and sera from COVID-19 PCR confirmed patients were used as controls to train a machine learning tool: support vector machine (SVM), Scikit learn [9] to generate an appropriate algorithm to classify serostatus. Full details are provided in Appendix and Figures 1.A and 1.B.

We were confident in our downstream analyses that seropositive samples are true positives due to the high sensitivity and specificity of our model as shown by Receiver Operator Curve (ROC) analysis (Figure 1.B). 
A a
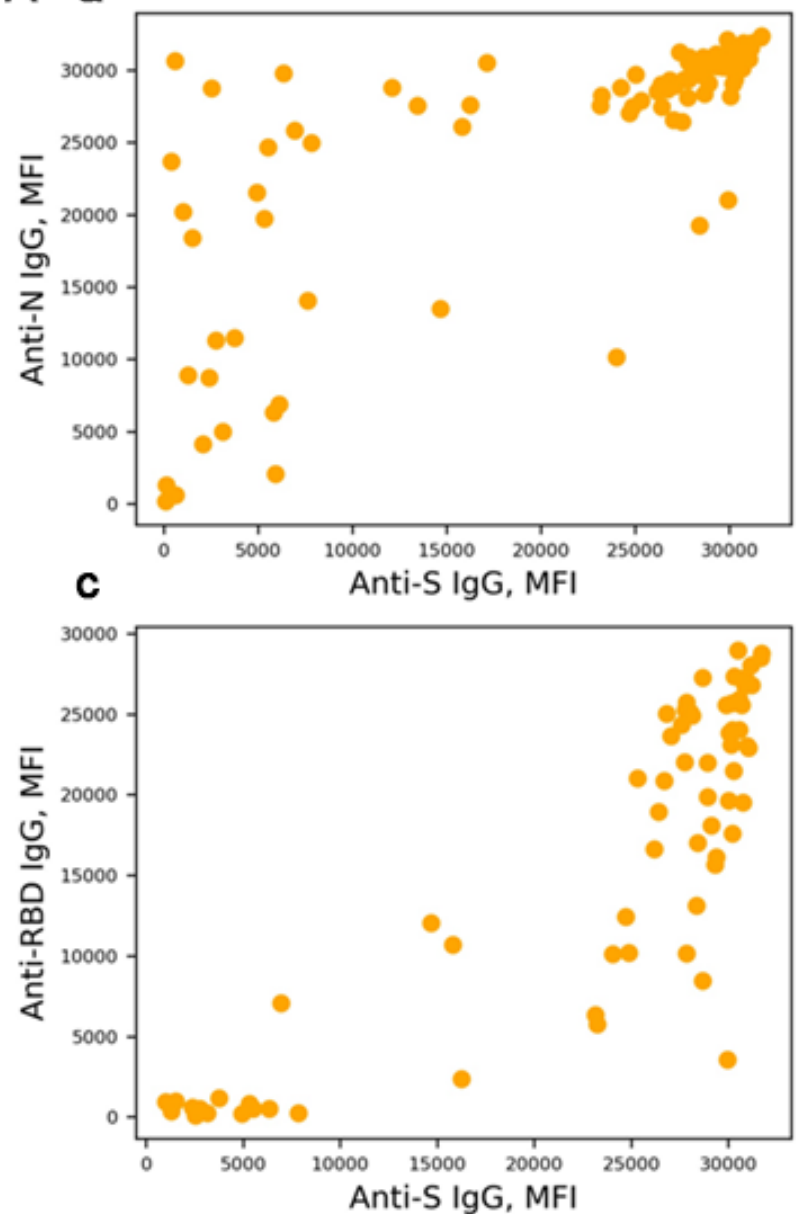

b
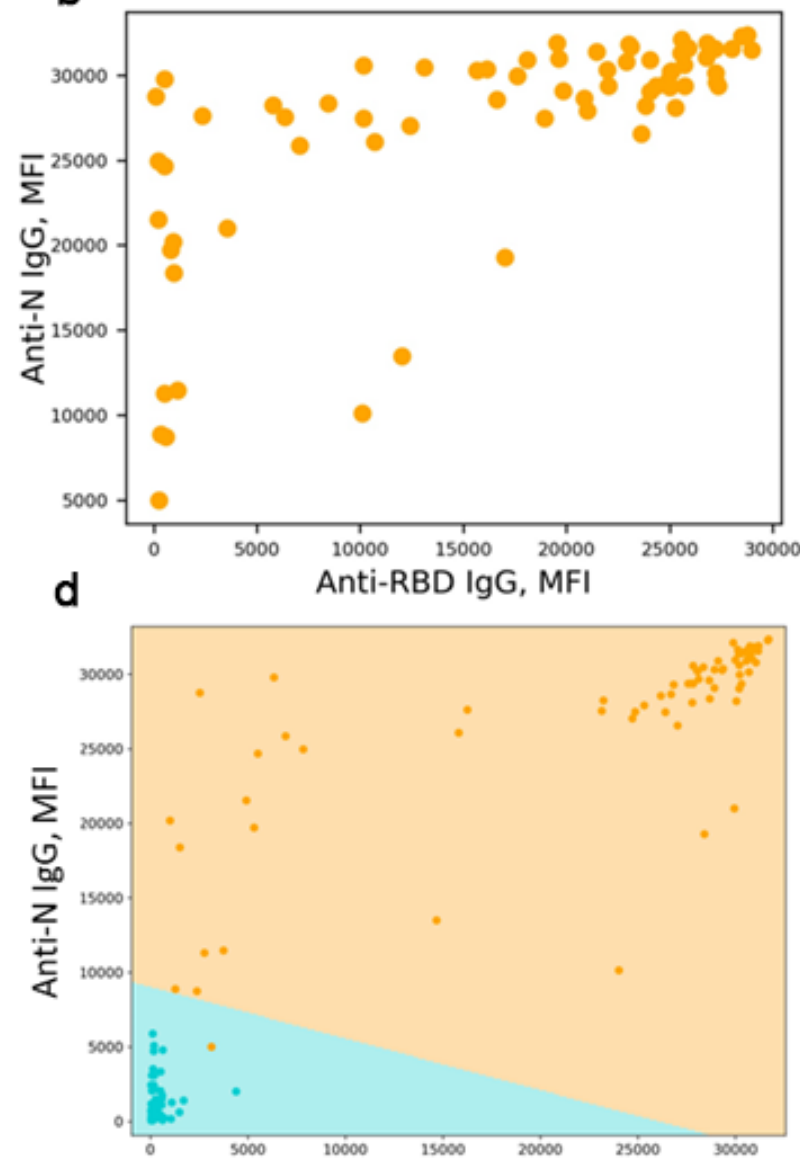

Anti-S IgG, MFI

\section{B ROC analysis}
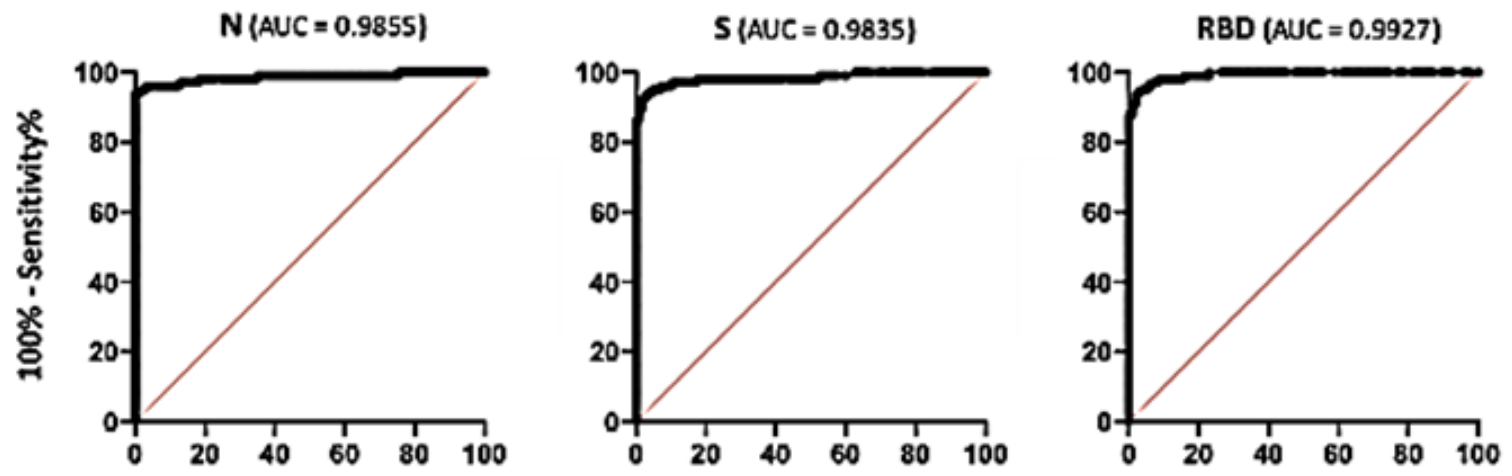

$100 \%$ - Specificity\%

Fig. 1. Training data set used in the support vector machine (SVM) and applied to classification of HCW serostatus and sensitivity/specificity analysis from PCR confirmed SARS-CoV-2 positive patients and pre-pandemic negative control samples are shown. A. Luminex values (MFI) show the relationship between (a) N and S, (b) N and RBD, (c) S and RBD in COVID-19 patients. (d) Illustrates how the SVM identifies cut off between HCW classified seropositive (orange) and seronegative (blue) samples taking into account the MFI binding to each antigen tested. The background colour indicates whether a sample at that location would be classified as seropositive (pale orange) or seronegative (pale blue). The classification is made based on all antigens at once, i.e. there is no single cut off point for any single antigen. The cut off shown here assumes the average pre-pandemic anti RBD response. For lower RBD responses the blue orange cut off is moved down and left. B. Receiver Operator Curves (ROC) for individual N, S and RBD antigens demonstrates the sensitivity and specificity of the Luminex assay with area under the curve (AUC) value embedded in each ROC analysis. 


\section{Statistical methods}

Binomial logistic regression was used to estimate probability of being seropositive by patient facing role and working location using $\mathrm{R}$ [10]. The significance was assessed by Wald Z-tests.

Proportional odds logistic regression models were used in R using the MASS package to understand the relationship between age, sex, and infection severity [11].

Severity classes were 1 (asymptomatic), 2 (mild), 3-4 (moderate-severe) (class 3 and 4 were grouped together as only one member of staff reported severity 4 ). The effect of age was allowed to differ between the sexes and the significance was determined by likelihood ratio test. The predicted probabilities and their confidence intervals were visualised using the effects $R$ package $[12,13]$. The assumption of proportional odds was assessed by refitting the model as two binary logistic regressions and comparing the estimated coefficients.

\section{Symptoms of seropositive and seronegative staff}

Data were collected from staff on ten symptoms that have been associated with COVID19. Logistic regression analysis was used to assess whether any of the reported symptoms were discriminative for serostatus. To counteract inflated false positive rates, the pvalue cut off for significance was adjusted following the Benjamini-Hochberg procedure based on the 10 tests [14].

\section{RESULTS}

Results are reported for the first 500 of recruited staff members by age, sex, ethnicity and work location and serostatus (Table 1). The median age was 42 years, $70 \%$ of the staff recruited were women and $78 \%$ of white ethnicity. The majority of staff were patient facing (82\%) and $25 \%$ worked in critical care (including a number of staff providing interhospital COVID19 patient transfers (data not shown). Co-morbidities were reported in $22 \%$ of the cohort and included asthma, hypothyroidism, cardiovascular disease, diabetes and anxiety/ depression (data not shown).

\section{Serostatus classification}

Serum results were available for 498 staff. In total we classified 70 staff members seropositive and 428 seronegative based on $\mathrm{S}, \mathrm{RBD}$, and $\mathrm{N}$ binding using the SVM. There was strong correlation in binding intensity across the 3 antigens however binding profiles were diverse and there were some individuals having high binding to only $\mathrm{N}$ antigen and fewer to $\mathrm{S}$ and $\mathrm{RBD}$ or $\mathrm{RBD}$ alone (Figure 2).

As there were no samples in our training set with high RBD without similarly high $\mathrm{N}$ binding (Figure 1.A), samples with elevated RBD binding alone were classified as seronegative by our SVM. This highlights the diversity of serostatus and the importance of capturing that diversity when defining seropositivity and negativity.

Table 1. Cohort demographic for age, sex, ethnicity and work location and by serostatus by multiplex ( $\mathrm{N}=498 \mathrm{classified).}$ Values: $\mathrm{N}=$ (\% of total and of cohort by demographic classification to nearest whole number). Age: years. ${ }^{*} p=0.017:$ age of seropositive and seronegative cohort (MW-U).

\begin{tabular}{|c|c|c|c|c|}
\hline \multirow{2}{*}{\multicolumn{2}{|c|}{ Number recruited }} & \multirow{2}{*}{$\begin{array}{c}\text { Total (\%) } \\
500 \\
\end{array}$} & \multirow{2}{*}{$\begin{array}{c}\text { Seropositive (\%) } \\
70(14)\end{array}$} & \multirow{2}{*}{$\begin{array}{c}\text { Seronegative (\%) } \\
428(86)\end{array}$} \\
\hline & & & & \\
\hline \multicolumn{2}{|c|}{ Age (yrs, median with IQR) } & $42(33-51)$ & $40(32-50)$ & $47(36-53)^{*}$ \\
\hline \multirow[t]{2}{*}{ Sex } & male & $146(29)$ & $20(14)$ & $126(86)$ \\
\hline & female & $352(70)$ & $50(14)$ & $302(86)$ \\
\hline \multirow[t]{6}{*}{ Ethnicity group } & 1:white & $390(78)$ & $45(12)$ & $345(88)$ \\
\hline & 2:mixed & $16(3)$ & $4(25)$ & $12(75)$ \\
\hline & 3:asian & $79(16)$ & $16(20)$ & $63(80)$ \\
\hline & 4:black & $11(2)$ & $2(18)$ & $9(82)$ \\
\hline & 5: other & $1(0.2)$ & 0 & $1(0.2)$ \\
\hline & NA & $3(1)$ & 0 & \\
\hline \multirow[t]{4}{*}{ Work location } & Critical Care patient facing & $126(25)$ & $10(7.9)$ & $116(92)$ \\
\hline & Non-Critical Care patient facing & $284(57)$ & $40(14)$ & $244(86)$ \\
\hline & Non-patient facing & $63(13)$ & $12(19)$ & $51(81)$ \\
\hline & NA & $27(5)$ & & \\
\hline
\end{tabular}


A
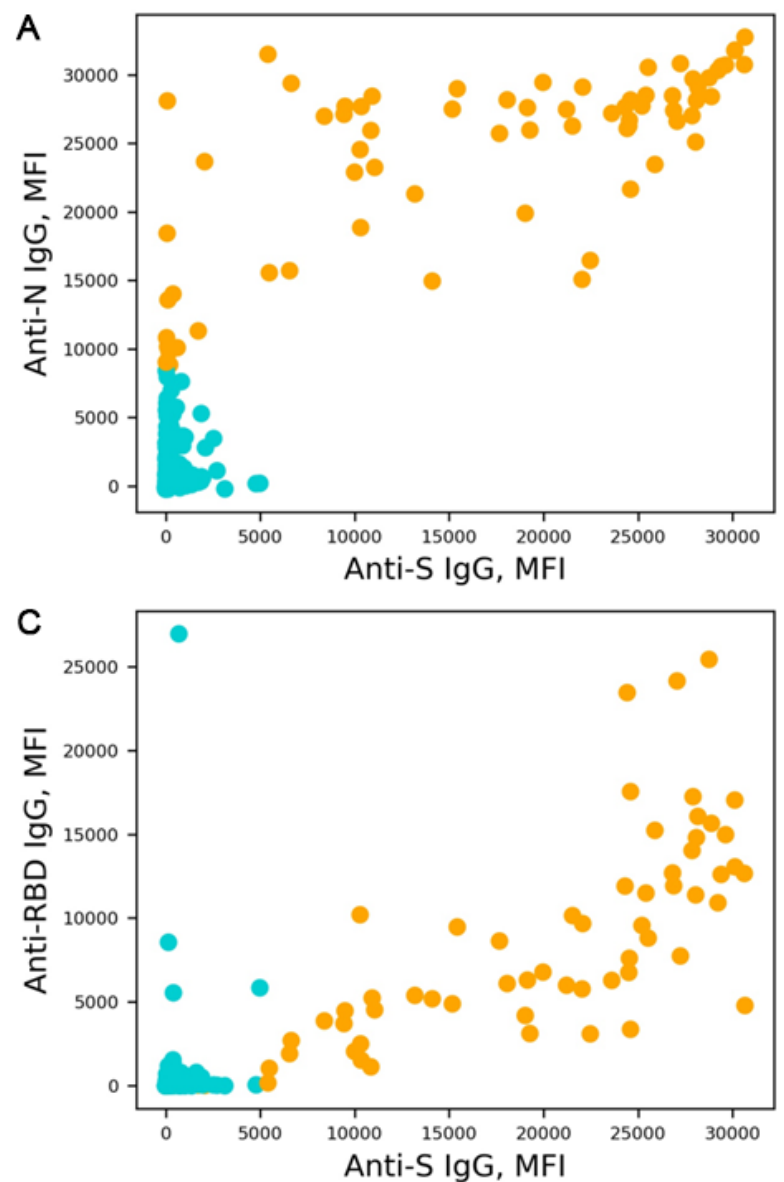
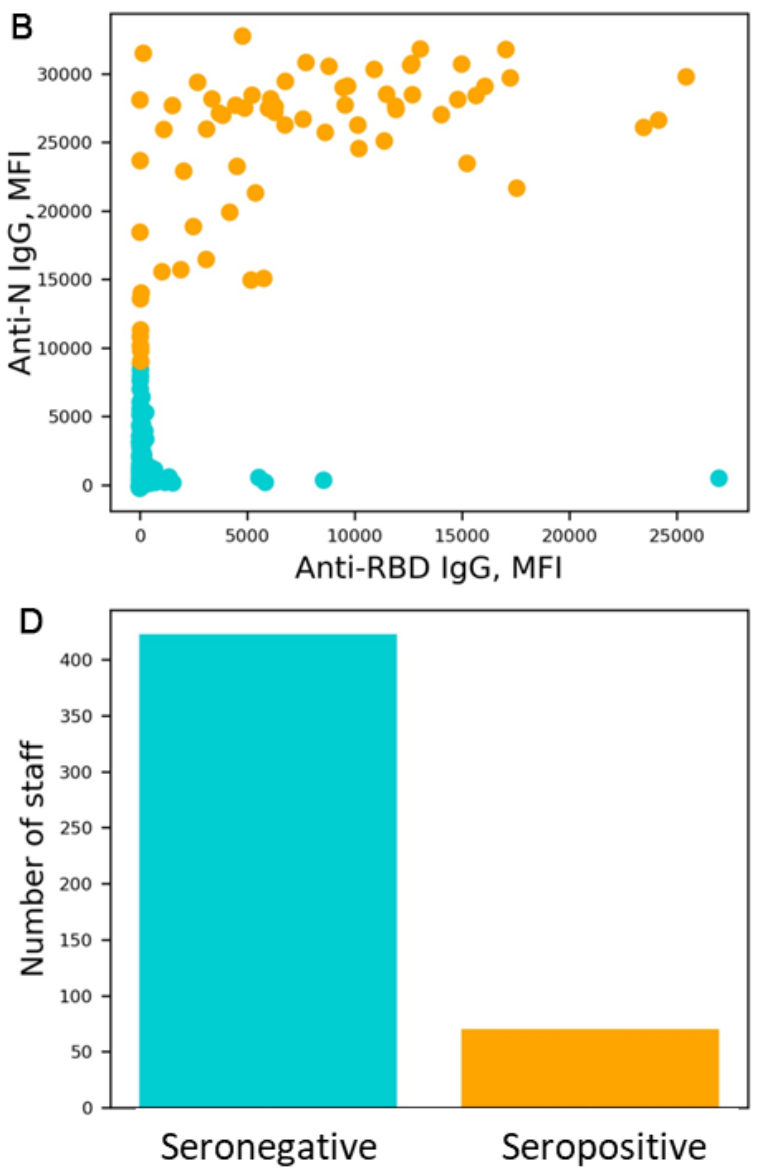

Fig. 2. Serostatus Classification. The mean fluorescent intensity (MFI) by Luminex of staff serum IgG binding to SARSCoV-2 proteins N, S and RBD, showing the relationship of binding between the three proteins : (A) N and S, (B) S-RBD and $\mathrm{N}$, (C) $\mathrm{S}$ and S-RBD. (D) shows the number of staff classified as seropositive or seronegative by the trained SVM. Serostatus is indicated by colour in all four panels: blue: seronegative; orange: seropositive.

\section{Serostatus by demographic, clinical role and location}

Comparing the demographic of the staff by serostatus classification, the seropositive staff were younger. Staff of non-white ethnicity were proportionately more likely to be seropositive (Table 1). There was no difference in the overall proportion of men and women classified seropositive.

Patient facing critical care staff were significantly less likely to be seropositive than staff in non-patient facing roles. $(\beta=-1.06, \mathrm{SE}=0.44, \mathrm{Z}=-2.39, \mathrm{p}=0.017$ ) (Table 1 , Figure 3).

Serostatus for non-critical care patient facing staff was not significantly different compared to non-patient facing staff $(\beta=-0.49, \mathrm{SE}=0.36, \mathrm{Z}=-1.14, \mathrm{p}=0.172)$.

\section{Serostatus by Symptom reporting and PcR diagnosis}

A high proportion of staff (45\%) reported possible COVID19 symptoms in the 4 months prior to recruitment and these were generally mild (Table 2).

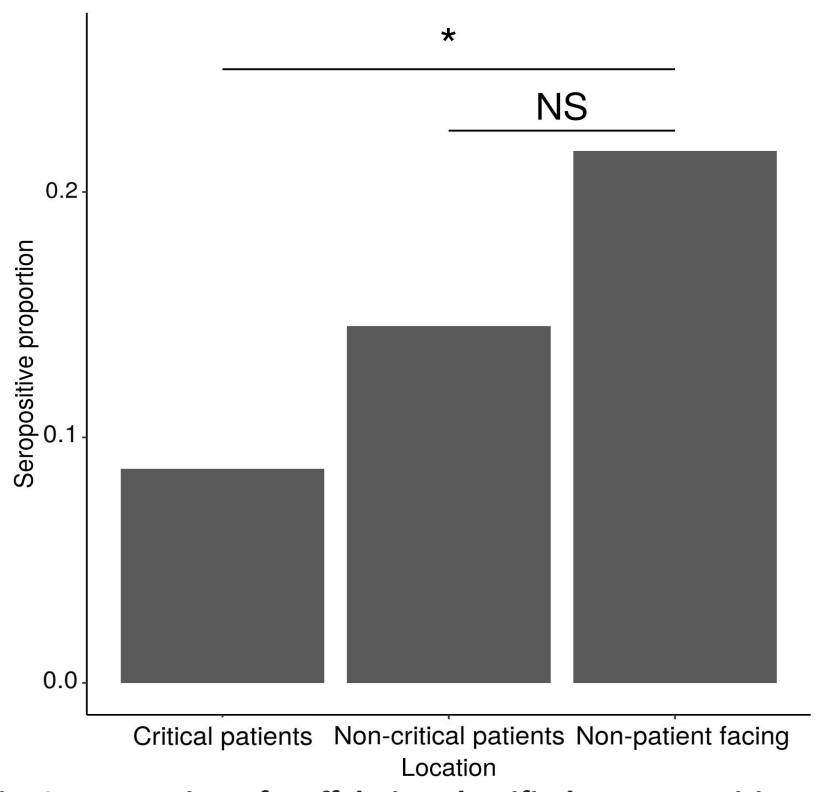

Fig. 3. Proportion of staff being classified as seropositive based on working location. (Proportion determined by Binomial regression. Significance determined by Wald-Z test. $* p=0.017$. 
Table 2. PCR Swab Positive and Symptom Severity Score by Serostatus. $N=$ (\% of total and of each cohort by severity score).

\begin{tabular}{|c|c|c|c|c|}
\hline & & Total & Seropositive & Seronegative \\
\hline PCR positive nasal swab & & $12(2)$ & $9(75)$ & $3(25)$ \\
\hline \multirow{5}{*}{ Symptom Severity Score } & 1: asymptomatic & $275(55)$ & $17(6)$ & 258 (94) \\
\hline & 2: mild & 195 (39) & $39(20)$ & $156(80)$ \\
\hline & 3: moderate & $26(5)$ & $9(35)$ & $17(65)$ \\
\hline & 4: severe & $1(0.2)$ & 1 & 0 \\
\hline & NA & $3(0.6)$ & & \\
\hline
\end{tabular}

The majority of these staff were found to be seronegative. Seropositive staff were proportionately more likely to describe moderate or severe symptoms and the single seropositive staff reporting severe symptoms had a history of severe cardiovascular disease. To determine whether certain symptoms were discriminating for serostatus, we looked at specific symptom reporting by serostatus (Figure 4.)

The only symptoms which could significantly predict serostatus were anosmia $(\mathrm{p}<0.001)$ and fever $(\mathrm{p}=0.031)$. Myalgia was also tested but was non-significant $(\mathrm{p}=0.301)$. However, after accounting for multiple testing only anosmia remained a significant predictor of serostatus. We found no relationship between symptom score and the spectrum of binding activity to $\mathrm{N}, \mathrm{S}$ and RBD proteins (data not shown).

Of the few staff who had PCR swab results from time of symptoms $(\mathrm{N}=10), 2$ (both reporting moderate severity symptoms) were seronegative in convalescence.

Analysis of symptom severity in the seropositive cohort indicated that male staff's risk of severe infection rose steeply with age but age had little effect on the risk of severe infection among female staff (age $\beta=-0.00$, $\mathrm{SE}=0.03$, sex male $\beta=-4.53$, age:sex male $\beta=0.13)$. The intercept for asymptomatic cases was $-0.85(\mathrm{SE}=1.25)$ and for severity class 2 the intercept was $2.11(\mathrm{SE}=1.30)$.

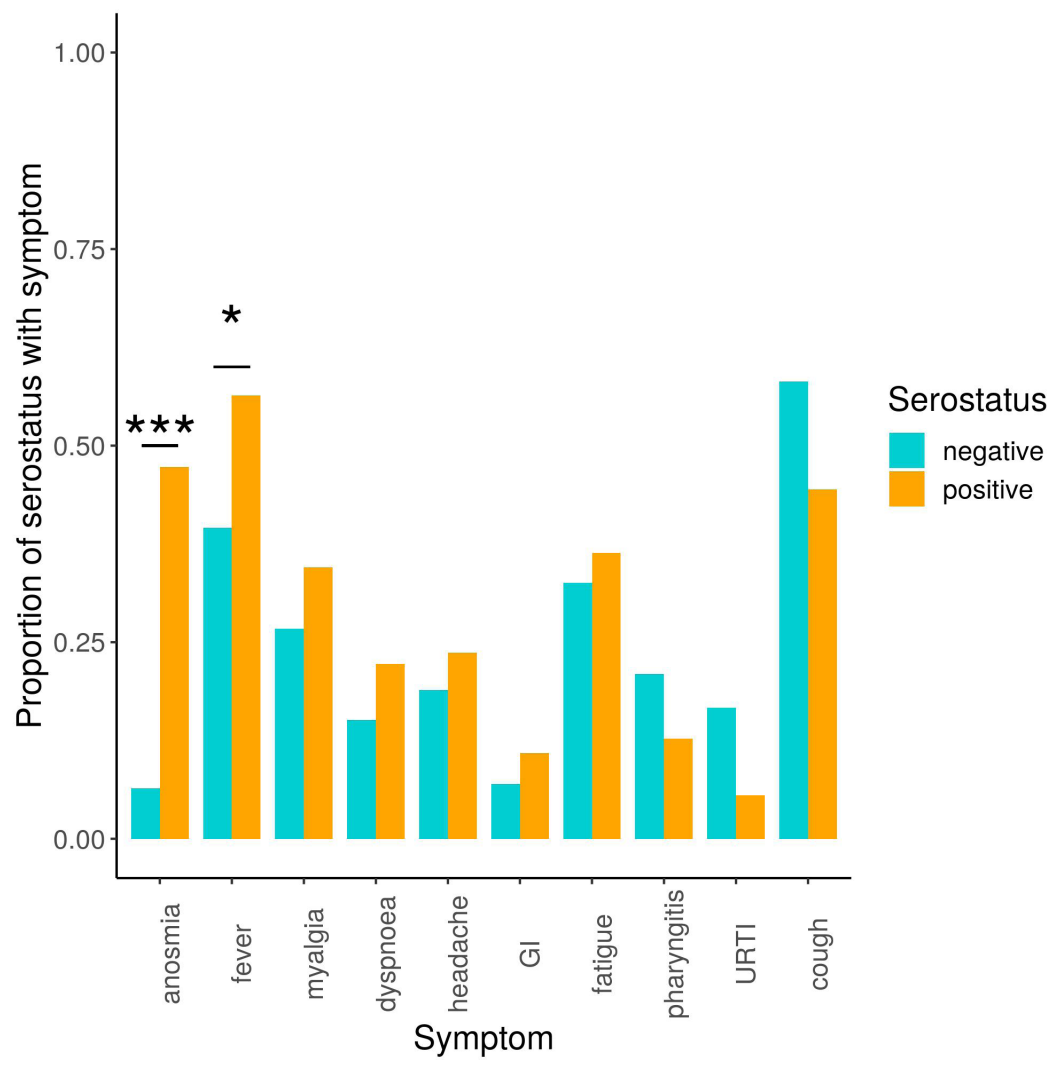

Fig. 4. Proportion of seronegative and seropositive staff reporting specific symptoms. Bars in orange indicate the proportion of seropositive staff with a symptom while bars in turquoise indicate seronegative staff proportions. Symptoms are ordered by difference in proportion between seropositive and seronegative staff. $* * \mathrm{p}=0.031 ; * * * \mathrm{p}<0.001$. 
Available online at: www.jccm.ro

The effect of age appears to be driven by male cases as when a model was fit to only female staff an age effect was not significant $(-2 \mathrm{LL}=0.02, \mathrm{p}=0.89)$. (Figure 5.a and 5.b).
The Journal of Critical Care Medicine 2021;7(3) • 205

The study was not powered to determine whether co-morbidities by sex may account for this difference. There was no relationship between age or sex and symptom severity for seronegative staff.
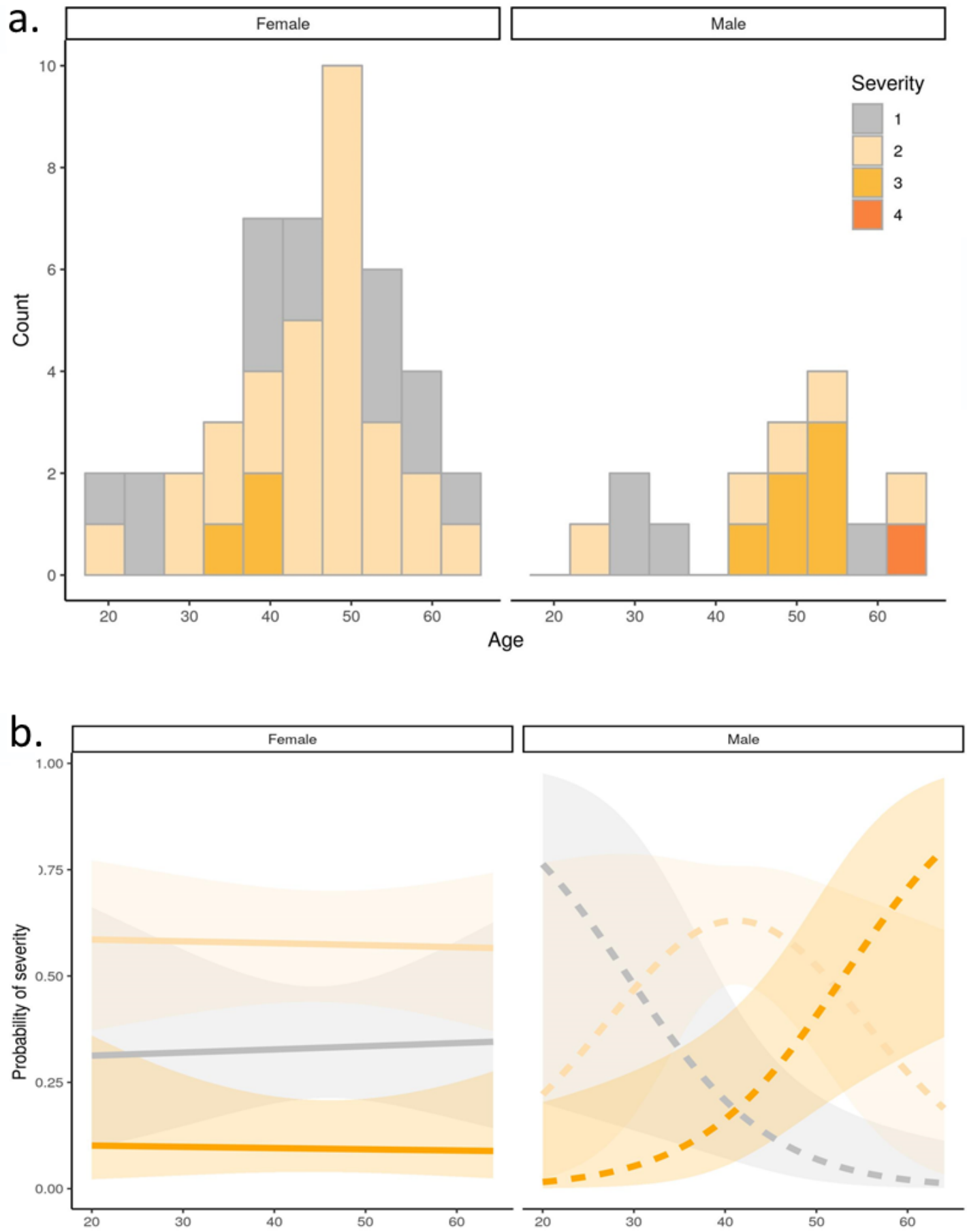

Age (Years)

Fig. 5. Symptom severity score by age and sex. a. Stacked histogram of seropositive staff age split by sex and coloured by severity of infection. b. Probability of disease severity based on age and sex of seropositive staff. Severity of infection is indicated by colour; grey is class 1 (asymptomatic), pale orange is class 2, and dark orange is class 3 or 4 (grouped due to small sample size). 


\section{DISCUSSION}

Through developing and applying a multiplex platform to simultaneously assess serum binding to multiple antigens, we demonstrate that staff working with COVID-19 patients in a critical care environment have a lower prevalence of serum antibodies to SARS-CoV-2 than those working in non-critical care and non-patient facing environments. It is unclear if differences in seroprevalence between staff groups represent risk of workplace infection or whether non-work place risk factors such as sociodemographic and lifestyle account for this. However at the time of the study, most non-clinical facing staff worked in open plan offices where the wearing of face masks was not policy at that stage and social distancing was inconsistently adhered to. In contrast, staff in critical care adhered to guidance strictly. These results may provide re-assurance to critical care staff and staff running hospital transfers including ECMO implementation, that the current PPE guidelines are fit for purpose. For staff in non-patient facing environments, prevalence of exposure was high suggesting that nosocomial infection risk may be higher than anticipated and stringent infection control measures are likely indicated. A potential confounder to this interpretation is that the participants in the study were self-selected and as such this may introduce bias however these results complement those of other published studies, showing that staff working in the critical care environment are at lower risk of workplace acquired infection compared with other health care environments [15]. Whether all hospital workers are at increased risk of COVID-19 is unclear as demographic matching is required to answer this question. At the time of staff sampling, regional seroprevalence was reported to be approximately $8 \%$ [16], considerably lower than the staff cohort assessed.

Nearly $50 \%$ of the staff recruited reported a history of symptoms that they felt may have represented SARSCoV-2 infection in the preceding 1-4 months, however $14 \%$ of staff were found to be seropositive. With considerable overlap between symptoms reported in COVID-19 and from infection with other common respiratory viruses, identifying symptoms that are more specific to SARS-CoV-2 infection is important, not least as PCR based screening resources even in the most wealthy countries are limited to allow targeted screening of individuals presenting with symptoms most likely representing COVID19 infection.

Analysis of free field symptom reporting, and correcting for multiple tests, we found that anosmia was the most discriminating symptom for seropositive status in our cohort. This is consistent with recently published reports of COVID-19 confirmed patients reporting anosmia additional to fever and continuous cough as being one of the most discriminating symptoms of COVID-19 [17-19]. Recent onset anosmia has now been added to the 3 symptoms used to diagnose COVID-19 and prioritise track and trace screening in the UK [20]. To allow distinction from other respiratory viruses, continuous evaluation of these three symptoms (anosmia, persistent cough and fever) to direct COVID-19 testing will be important noting that in our cohort, $25 \%$ of seropositive staff reported no history of symptoms that they considered consistent with possible COVID-19. These results complement existing data generated through both seroprevalence and virus surveillance in health care workers and the population, demonstrating that $15-40 \%$ of individuals have asymptomatic infection in the UK with highest prevalence in younger cohorts $[15,19,21]$.

More detailed interpretation of these data is limited by the low numbers of staff with contemporaneous PCR swab results as staff were advised to self-isolate based on symptom reporting without PCR based confirmation. Of the 12 staff who reported previous SARSCoV-2 PCR positive by nasal swabbing (10 symptomatic, 2 though surveillance), 3 were seronegative (all female and 2 moderately symptomatic at time of swabbing). Serum samples for all staff were taken at least 4 weeks after symptom reporting so this does not reflect premature serum sampling rather it suggests either that seroprevalence under-represents infection rates or that PCR were false positive [22]. Diagnostic thresholds for the serology assay we used were established using acute phase sera from patients with moderate to severe disease and as such may have reduced sensitivity for detecting history of exposure particularly in those with asymptomatic or mild infection in whom short lived or no seroconversion has been reported [23].

COVID-19 Symptom severity scoring showed a striking relationship between age and symptom severity in seropositive male staff. There was no relationship between age and symptom severity in female seropositive staff or seronegative staff. Being of male sex and aged $>40$ years are reported risk factors for severe COVID-19 and data is now emerging suggesting the immune response to the virus differs between sexes likely contributing to the differences in nature and severity of disease between men and women [24]. Older 
males are at increased risk of pneumonic complications from other viruses such as influenza [25] and other causes of community acquired pneumonia [26] however on multivariate analysis the male sex as an independent predictor of disease severity is often not sustained. Detailed analysis of the relationship between age and sex in seasonal and pandemic influenza has shown that sex hormones and virus specific pathogenesis of disease influence the relationship between age, sex and disease severity in man and in murine models of disease [27]. Whether this was relevant in the cohort presented is not known noting that there are many confounding variables including BMI and co-morbidities that may influence disease severity which this study was not powered to explore. However understanding the differences between the sexes in pathogenesis (and possibly immunopathogenesis, including antibody mediated exacerbation) of COVID-19 will be important to inform targeted disease prevention and treatment strategies.

Whether individuals with PCR confirmed infection who do not seroconvert are at increased risk of re-infection compared with those who develop high serum antibody titres remains an open question. This unknown generates considerable anxiety for staff. Despite widely published concerns relating to an 'immunity passport', staff are generally self-re-assured that if they develop antibodies post infection that they may be less at risk of subsequent re-infection. Some exposed individuals have been reported to have evidence of cell mediated immune responses in the absence of serum antibodies [28]. How prevalent this is and whether a cell mediated response in the absence of serum antibody protects from re-infection is not yet known.

This study has a number of limitations. Most significantly, nasal swabbing for SARS-CoV-2 PCR tests were not available for symptomatic staff early in the pandemic. when most of our staff reported symptoms. Some care should be taken when interpreting the exact relationship between severity of infection and age of men as relatively few male staff members were classified as seropositive. However, many other studies have similarly reported increased incidence of severe COVID-19, especially in older men [29-31].

Large cohort, longitudinal studies with paired swab and serum samples additional to symptom reporting are now running. In the UK, the Sarscov2 Immunity \& REinfection EvaluatioN longitudinal health care worker surveillance study, SIREN [32] is underway. Swab and serum samples are collected at 2-4 weekly intervals in large cohorts, in addition to symptom reporting. This will provide the power to define in detail the relationship between serum response, symptom severity and re-infection risk in HCW by demographic. Although it is important to acknowledge that many staff identified as being at increased risk of severe COVID-19 have been shielding and/or working remotely and may be under-represented in these workplace based cohort studies.

In conclusion, we have shown that staff working in this critical care environment looking after large numbers of COVID-19 patients including the transfer of acutely unwell patients for escalation of care, have no serological evidence of increased SARS-CoV-2 exposure compared with staff in non-clinical roles. Of the symptoms reported, anosmia most reliably predicts seropositive infection in this cohort. Severity of symptoms increases by age in male and not female HCWs and the reason for this remains unclear.

\section{ABBREVIATIONS}

COVID-19: coronavirus disease 2019

SARS-CoV-2: severe acute respiratory syndrome coronavirus 2

HCW: Health Care Workers

PCR: polymerase chain reaction

PPE: Personal Protective Equipment

\section{ACKNOWLEDGEMENTS}

Royal Papworth Hospital NHS Trust: R\&D pump priming. This work is now funded by the UKRI and NIHR MC_PC_20016: HICC: Humoral Immune Correlates for COVID19: Defining protective responses and critical readouts for Clinical Trials of Vaccines and Therapeutics. We would like to thank staff from RPH recruited to the study, Dr Ian Smith ${ }^{1}$, Professor William Schwaeble and Dr Javier Castillo-Olivares Pallardo ${ }^{2}$ for critical review of the manuscript and Leo Kiss of MRC LMB for helping with SARS-CoV-2 $\mathrm{N}$ protein reagents. MP was supported by the Jane and Aatos Erkko Foundation and Orion Research Foundation sr.

\section{- AUTHOR CONTRIBUTIONS}

HEB and JLH conceived the project and planned the study. HEB secured ethical approval. HEB, DW and 
JLH secured funding. Recruitment of participants, data management and sera collection were performed by HEB and JG. Sera processing was performed by RD, AN, MP, PT and GC. JAGB, XX, JAN, GLG, LCJ and JL generated and provided the SARS-CoV-2 proteins to support $\mathrm{RD}$ and $\mathrm{SL}$ in establishing and validating the luminex platform. RD, SL, AS, LC-G and SE analysed the sera by luminex and reported the results. HEB, DW and $\mathrm{RD}$ analysed the data. HEB generated the tables; RD and DW generated the Figures. DW ran the statistical analysis. HEB, DW and RD wrote the manuscript with contribution from all authors.

\section{CONFLICT OF INTEREST}

None to declare.

\section{REFERENCES}

1. Zhu N, Zhang D, Wang $W$ et al. A Novel Coronavirus from Patients with Pneumonia in China, 2019. N Engl J Med. 2020;382(8):727-33.

2. WHO. Coronavirus disease (COVID-19) Weekly Epidemiological Update and Weekly Operational Update (available from: https:// www.who.int/emergencies/diseases/novel-coronavirus-2019/ situation-reports)

3. Zheng S, Fan J, Yu F et al. Viral load dynamics and disease severity in patients infected with SARS-CoV-2 in Zhejiang province, China, January-March 2020: retrospective cohort study. BMJ. 2020;369:m1443.

4. Shah ASV, Wood R, Gribben C et al. Risk of hospital admission with coronavirus disease 2019 in healthcare workers and their households: nationwide linkage cohort study. BMJ. 2020 Oct $28 ; 371$.

5. Milner A, BakerE, JerajS et al. Race-ethnic and gender differences in representation within the English National Health Service: a quantitative analysis. BMJ Open. 2020;10(2):e034258.

6. Williamson EJ, Walker AJ, Bhaskaran $\mathrm{K}$ et al. Factors associated with COVID-19-related death using OpenSAFELY. Nature. 2020;584(7821):430-6.

7. Abbas A, Memon SF, Khattab N et al. COVID-19 risk assessments: shortcomings in the protection of Black, Asian and Minority Ethnic healthcare workers. J Hosp Infect. 2020;106(2):385-6.

8. WHO. Clinical management of COVID-19. Interim Guidance 2020. (available at: https://www.who.int/publications/i/item/ clinical-management-of-covid-19).

9. Pedregosa F, Varoquaux G, Gramfort A, et al. Journal of Machine Learning Research (2011) 12. 2825-2830.

10. R: A language and environment for statistical computing. $R$ Foundation for Statistical Computing, Vienna, Austria. R Core Team (2018). (Available online at https://www.R-project.org/.)
11. Venables WN and Ripley. B.D. Modern Applied Statistics with S. Springer-Verlag. New York 2002.

12. Fox J, Weisberg S. An R Companion to Applied Regression.3 ed. $\therefore$ Sage, Thousand Oaks CA. 2019

13. Fox J, Hong J. Effect Displays in R for Multinomial and Proportional-Odds Logit Models: Extensions to the effects Package. Journal of Statistical Software, 32(1), 1-242009.

14. Benjamini Y, Hochberg Y. Controlling the False Discovery Rate: A Practical and Powerful Approach to Multiple Testing. Journal of the Royal Statistical Society Series B (Methodological). 1995;57(1):289-300.

15. Shields A, Faustini SE, Perez-Toledo $M$ et al. SARS-CoV-2 seroprevalence and asymptomatic viral carriage in healthcare workers: a cross-sectional study. Thorax. 2020. Dec;75(12):1089-1094.

16. England PH. Weekly Coronavirus Disease 2019 (COVID-19) Surveillance Report. Summary of COVID-19 surveillance systems 2020. https://www.gov.uk/government/news/weeklycovid-19-surveillance-report-published

17. Struyf T, Deeks JJ, Dinnes J et al. Signs and symptoms to determine if a patient presenting in primary care or hospital outpatient settings has COVID-19 disease. Cochrane Database Syst Rev. 2020;7:CD013665.

18. Hashmi HAS, Asif HM. Early Detection and Assessment of Covid-19. Frontiers in Medicine. 2020;7(311).

19. Gómez-Ochoa SA, Franco OH, Rojas LZ et al. COVID-19 in Health-Care Workers: A Living Systematic Review and MetaAnalysis of Prevalence, Risk Factors, Clinical Characteristics, and Outcomes. Am J Epidemiol. 2021 Jan 4;190(1):161-175.

20. Get a free NHS test to check if you have coronavirus. 2020. (Available online at: https://www.gov.uk/get-coronavirus-test).

21. Pollán M, Pérez-Gómez B, Pastor-Barriuso et al. Prevalence of SARS-CoV-2 in Spain (ENE-COVID): a nationwide, populationbased seroepidemiological study. Lancet. 2020 Aug 22;396(10250):535-544.

22. Surkova E, Nikolayevskyy V, Drobniewski F. False-positive COVID-19 results: hidden problems and costs. Lancet Respir Med. 2020 Dec;8(12):1167-1168.

23. Seow J, Graham C, Merrick B et al. Longitudinal observation and decline of neutralizing antibody responses in the three months following SARS-CoV-2 infection in humans. Nat Microbiol. 2020 Dec;5(12):1598-1607.

24. Takahashi T, Ellingson MK, Wong $P$ et al. Sex differences in immune responses that underlie COVID-19 disease outcomes. Nature. 2020 Dec;588(7837):315-320.

25. Mertz D, Kim TH, Johnstone Jet al. Populations at risk for severe or complicated influenza illness: systematic review and metaanalysis. BMJ. 2013 Aug 23;347.

26. Ishiguro T, Takayanagi N, Yamaguchi S, et al. Etiology and Factors Contributing to the Severity and Mortality of Communityacquired Pneumonia. Internal Medicine. 2013;52(3):317-24.

27.Vermillion MS, Ursin RL, Kuok DIT et al. Production of 
Available online at: www.jccm.ro

amphiregulin and recovery from influenza is greater in males than females. Biology of Sex Differences. 2018;9(1).

28. Sekine T, Perez-Potti A, Rivera-Ballesteros O et al. Robust T Cell Immunity in Convalescent Individuals with Asymptomatic or Mild COVID-19. Cell. 2020 Oct 1;183(1):158-168

29. Chen N, Zhou M, Dong X et al. Epidemiological and clinical characteristics of 99 cases of 2019 novel coronavirus pneumonia in Wuhan, China: a descriptive study. The Lancet. 2020;395(10223):507-13.

30. Knight SR, Ho A, Pius $R$ et al. Risk stratification of patients admitted to hospital with covid-19 using the ISARIC WHO
The Journal of Critical Care Medicine 2021;7(3) • 209

Clinical Characterisation Protocol: development and validation of the 4C Mortality Score. BMJ. 2020 Sep 9; 370.

31. Drefahl S, Wallace M, Mussino E et al. A population-based cohort study of socio-demographic risk factors for COVID-19 deaths in Sweden. Nature Communications. 2020; Oct 9;11(1):5097.

32. SIREN (Sarscov2 Immunity \& REinfection EvaluatioN): The impact of detectable anti SARS-COV2 antibody on the incidence of COVID-19 in healthcare workers. 2020. (https://www. hra.nhs.uk/planning-and-improving-research/applicationsummaries/research-summaries/siren-sars-cov2-immunityand-reinfection-evaluation-covid-19-uph/ ) 


\section{APPENDIX}

\section{SARS-CoV-2 serology by multiplex particle-based flow cytometry (Luminex)}

Recombinant SARS-CoV-2 N, S and RBD were covalently coupled to distinct carboxylated bead sets (Luminex; Netherlands) to form a 3-plex assay. The S protein construct used is S-R/PP as described in Xiong et al 2020 [1]. The RBD protein construct used is described by Stadlbauer et al [2]. The N protein used is a truncated construct of the SARS-CoV-2 N protein comprising residues 48-365 (both ordered domains with the native linker) with an $\mathrm{N}$ terminal uncleavable hexahistidine tag. N was expressed in E. Coli using autoinducing media for $7 \mathrm{~h}$ at $37^{\circ} \mathrm{C}$ and purified using immobilised metal affinity chromatography (IMAC), size exclusion and heparin chromatography. Beads were first activated with 1-ethyl-3-[3-dimethylaminopropyl]carbodiimide hydrochloride (Thermo Fisher Scientific) in the presence of N-hydroxysuccinimide (Thermo Fisher Scientific), according to the manufacturer's instructions, to form amine-reactive intermediates. The activated bead sets were incubated with the corresponding proteins at a concentration of $50 \mu \mathrm{g} / \mathrm{ml}$ in the reaction mixture for $3 \mathrm{~h}$ at room temperature on a rotator. Beads were washed and stored in a blocking buffer (10 mM PBS, $1 \%$ BSA, $0.05 \%$ NaN3). The N-, S- and RBD-coupled bead sets were incubated with proband sera at a $1 / 100$ dilution for $1 \mathrm{~h}$ in 96-well filter plates (MultiScreen HTS; Millipore) at room temperature in the dark on a horizontal shaker. Fluids were aspirated with a vacuum manifold and beads were washed three times with 10 $\mathrm{mM}$ PBS $/ 0.05 \%$ Tween 20 . Beads were incubated for 30 min with a PE-labeled anti-human IgG-Fc antibody (Leinco/Biotrend), washed as described above, and resuspended in $100 \mu \mathrm{PBS} /$ Tween. They were then analyzed on a Luminex analyzer (Luminex / R\&D Systems) using Exponent Software V31. Specific binding was reported as mean fluorescence intensities (MFI).

\section{Serostatus classification}

A linear support vector machine (SVM) in scikitlearn [3] was used to classify samples as seropositive or seronegative based on Luminex readings of SARSCoV-2 N, S, and S-RBD (Figure 1.A.a). Receiver operator analysis (ROC) was performed using GraphPadPrism8 software (Figure 1.B.) prior, to determine how useful the Luminex platform was at identifying SARSCoV-2 exposed individuals compared with non-exposed (pre-pandemic) individuals. The area under the curve (AUC) was over $98 \%$ for each antigen tested confirming high sensitivity and specificity of the assay in detecting SARS-CoV-2 immune exposure for these cohorts. 101 hospitalised patients with confirmed COVID-19 and 126 pre-pandemic control samples were included in the training set. Luminex readings were centred and scaled by the mean and standard deviation of the training set. To assess the accuracy of our SVM we used 5-fold cross validation, preserving the ratio of seropositive to seronegative cases. Then a model was trained on the whole training set and used to classify the Papworth staff cohort, after centering and scaling according to the training set mean(SD

The SVM was able to successfully classify most samples in our training set. During cross validation the average sensitivity and specificity were 0.97 and 1.0 respectively. We were thus confident in our downstream analyses that seropositive samples are true positives due to the high specificity of our model.

\section{- REFERENCES FOR APPENDIX}

1. Xiong X, Qu K, Ciazynska KA, et al. A thermostable, closed SARS-CoV-2 spike protein trimer. Nat Struct Mol Biol. Oct 2020; 27(10):934-941.

2. Stadlbauer D, Amanat F, Chromikova $V$, et al. SARS-CoV-2 Seroconversion in Humans: A Detailed Protocol for a Serological Assay, Antigen Production, and Test Setup. Curr Protoc Microbiol. 06 2020;57(1)

3 Pedregosa F, Varoquaux G, Gramfort A, et al. Journal of Machine Learning Research (2011) 12. 2825-2830. 